\title{
EMSx: a numerical benchmark for energy management systems
}

\author{
Adrien Le Franc ${ }^{* \dagger} \quad$ Pierre Carpentier ${ }^{\ddagger} \quad$ Jean-Philippe Chancelier* \\ Michel De Lara*
}

October 15, 2021

\begin{abstract}
Inserting renewable energy in the electric grid in a decentralized manner is a key challenge of the energy transition. However, at local scale, both production and demand display erratic behavior, which makes it challenging to match them. It is the goal of Energy Management Systems (EMS) to achieve such balance at least cost. We present EMSx, a numerical benchmark for testing control algorithms for the management of electric microgrids equipped with a photovoltaic unit and an energy storage system. EMSx is made of three key components: the EMSx dataset, provided by Schneider Electric, contains a diverse pool of realistic microgrids with a rich collection of historical observations and forecasts; the EMSx mathematical framework is an explicit description of the assessment of electric microgrid control techniques and algorithms; the EMSx software EMSx. $j 1$ is a package, implemented in the Julia language, which enables to easily implement a microgrid controller and to test it. All components of the benchmark are publicly available, so that other researchers willing to test controllers on EMSx may reproduce experiments easily. Eventually, we showcase the results of standard microgrid control methods, including Model Predictive Control, Open Loop Feedback Control and Stochastic Dynamic Programming.
\end{abstract}

Keywords Electric microgrid · Multistage stochastic optimization · Numerical benchmark

\section{Introduction}

Inserting renewable energy in the electric grid is a key challenge of the energy transition. As renewable power units are often coupled with a storage system and envisaged at a local scale (where the demand is more erratic than at a global scale), this makes the management of such electric microgrids delicate. In this paper, we concentrate on Energy Management Systems (EMS), a high level layer of hierarchical control, responsible for operating electric microgrids while optimizing security and economic criteria 15 .

The design of an EMS for the optimal management of a storage system supporting renewable energy integration is a well-known challenge. Most approaches are based on the mathematical theory of multistage deterministic and stochastic optimization, and the relevance of a microgrid control method is usually assessed on a representative validation setup.

* CERMICS, École des Ponts ParisTech, France.

${ }^{\dagger}$ Efficacity

${ }^{\ddagger}$ UMA ENSTA Paris 
A comparative study of EMS design techniques was conducted in [17], which includes Model Predictive Control (MPC, [3.7]), Open Loop Feedback Control (OLFC, [2, Vol. 1, §6.2], sometimes referred to as stochastic MPC) and Stochastic Dynamic Programming (SDP [1, 16]). Although providing a large benchmark of control methods, [17 assesses management strategies on a very specific example, the control of a braking energy storage system in a subway station. Another example of highly specific EMS assessment result is found in [13], where the authors apply MPC for operating a very detailed residential microgrid configuration, mixing thermal and electric energy storage, and providing models for every electric device accounting for a shiftable load in a standard Swiss household. Due to the specificity of the case studies, it is not clear that the conclusions of such works can be generalized, and that they can help researchers and practitioners willing to deploy an EMS for a new context, with new data.

Novel microgrid controller techniques are published regularly, making it even harder to establish an up-to-date benchmark. In [10], the authors propose an innovative EMS architecture which combines Recurrent Neural Networks 8] with Stochastic Dual Dynamic Programming [18 for managing solar panels coupled with a battery. They compare their EMS against a heuristic approach on residential data collected by a research organization which provides free access to its data for academic purposes. However, their simulation relies on a commercialized real-time simulator — which may improve the realism of the assessment method, but complicates the reproduction of the testbed. A similar microgrid application is considered in [11], where the authors also operate a battery for photovoltaic power integration in a novel framework, modeling the electric load by a stochastic differential equation. They rely on real data - whose access is not documented - from an experimental site in Chile, and implement their simulation on an open source numerical solver. The same initiative was taken in [9], where the authors make their implementation of SDP publicly available, so that their comparison with a heuristic method for the design of an EMS could be extended to other data and other techniques.

It is in that context that we introduce the EMSx benchmark, composed of three constituents - a dataset, a mathematical framework, and a simulation software - and designed for the purpose of assessing electric microgrid controllers on an open, transparent and unified challenge.

The first component of the EMSx benchmark is a new dataset reporting a wide range of contrasted microgrid situations. For these data, we rely on samples collected by the Schneider Electric (SE) company on 70 industrial sites. SE develops and commercializes microgrid controllers, and is interested in challenging its current practices with state-of-the art optimization methods developed in the academic world. In this context, SE puts together a dataset which allows benchmarking different energy management methods in various situations. This dataset contains photovoltaic and electric load profiles, detailing both historical observations and forecasts, which let us closely reproduce the online information available to an EMS.

The second component of the EMSx benchmark is a mathematical framework for the assessment of electric microgrid control techniques and algorithms. This framework consists of the mathematical description of a microgrid architecture - made of one resource, one battery, one load - and of an economic criterion that depends on how the battery is managed. Indeed, as we focus on photovoltaic units integrated in local power networks - with uncertainties arising both from the electric load and from the photovoltaic power generation - introducing an energy storage system can help to reduce the energy bill. The battery is managed by means of a microgrid controller, for which we propose a precise mathematical definition and a score 
to measure its aggregated performance, on the one hand across the uncertainties unique to a site, on the other hand across the different sites of the dataset.

The third component of the EMSx benchmark is the EMSx benchmark software, that we have developed and implemented as a Julia [4] package. This software makes possible the simulation and assessment of a large range of controllers on the testbed defined by our dataset and our mathematical framework.

We believe that our benchmark is well-suited for assessing the performance of a large class of control techniques, and we illustrate our claim by measuring the performance of a selection of controllers - derived from MPC, OLFC, SDP, and from an extended state formulation of a plain SDP controller that models uncertainties with an auto-regressive process (SDPAR, [19, §3.1.1] and [14]). All in all, EMSx stands out by providing open access to our simulation data and software, permitting to benchmark microgrid controllers on simulation data representative of a large panel of industrial microgrid cases, within a clear and detailed mathematical framework.

This paper is structured as follows. We introduce the EMSx benchmark dataset in Sect. 2 , the EMSx benchmark mathematical framework in Sect. 3 - by providing a microgrid simulation model, a definition of a microgrid controller and a score - and the EMSx benchmark EMSx.jl package in Sect. 4. In Sect. 5, we detail two main classes of mathematical techniques to design microgrid controllers, and we provide the numerical results obtained with the EMSx benchmark. The Appendix A gathers additional material on the numerical experiments of Sect. 5.

\section{The EMSx benchmark dataset}

We present the Schneider Electric (SE) dataset which offers a large collection of field data for studying the management of electric microgrids. The dataset is publicly available at URL https://github.com/adrien-le-franc/EMSx.jl.

In 82.1 , we present generalities about Schneider Electric's 70 sites. Then, we detail the content of the dataset by focusing on historical observations in 2.2 and on historical forecasts in $\$ 2.3$. Finally, in $\$ 2.4$, we illustrate how the 70 sites differ in terms of "predictability".

\subsection{Generalities about Schneider Electric's 70 sites}

Indeed, SE has collected a large database of load profiles on real operated microgrids deployed on a various collection of 70 industrial sites, mainly located in Europe and in the United States (for data privacy reasons, SE does not provide specific information on the origin of the sites).

Each site is documented with parameters and time series data. We denote the set of sites by $I$ (hence $|I|=70$ ). On each site $i \in I$, we provide battery parameters $\left(c^{i}, \bar{l}^{i}, \rho_{c}^{i}, \rho_{d}^{i}\right.$ ) (see the storage dynamics part in $\$ 3.1$ below). Regarding time series, the database contains historical observations and historical forecasts of the energy demand and of photovoltaic generation for each site. For this latter, however, SE has selected a single photovoltaic profile from a site

located in South Central United States, and has then rescaled this profile for each site of the database, taking care to restore the balance between the energy generation and the load profile. This special treatment of photovoltaic generation is due to the lack of detailed historical meteorological data for most of the sites, which is an impediment to compute accurate photovoltaic forecasts. 
We sample the continuous time every 15 minutes, giving, for each site $i \in I$, a time index $t \in\left\{1,2, \ldots, \theta^{i}-1, \theta^{i}\right\}$, up to the horizon $\theta^{i}$ (with at least one year of historical observations and forecasts per site). Every time interval $[t, t+1$ [ corresponds to 15 minutes.

\subsection{Historical observations}

We have measures of the photovoltaic generation $g_{t}$ and of the energy demand $d_{t}$ over the last 15 minutes, providing vectors of historical observations for each site $i \in I$ :

$$
\begin{aligned}
& g^{i}=\left(g_{1}^{i}, \ldots, g_{\theta^{i}}^{i}\right) \in \mathbb{R}^{\theta^{i}}, \quad \forall i \in I, \\
& d^{i}=\left(d_{1}^{i}, \ldots, d_{\theta^{i}}^{i}\right) \in \mathbb{R}^{\theta^{i}}, \quad \forall i \in I .
\end{aligned}
$$

We provide examples of observed daily chronicles for photovoltaic generation in Figure 1, and for energy demand in Figure 2.



Figure 1: Examples of daily chronicles (historical observations) of photovoltaic generation (1a) (from Site 25).



Figure 2: Examples of daily chronicles (historical observations) of energy demand (1b) (from Site 25)

\subsection{Historical forecasts}

On top of observed data, Schneider Electric also provides, every 15 minutes, historical forecasts $\hat{g}_{t, t+1}^{i}, \ldots, \hat{g}_{t, t+96}^{i}$ of photovoltaic profiles and historical forecasts $\hat{d}_{t, t+1}^{i}, \ldots, \hat{d}_{t, t+96}^{i}$ of demand profiles for the next 24 hours (hence $96=24 \times 60 / 15$ ), hence giving vectors for all sites $i \in I$ and for all times $t \in\left\{1, \ldots, \theta^{i}\right\}$

$$
\begin{aligned}
& \hat{g}_{t}^{i}=\left(\hat{g}_{t, t+1}^{i}, \ldots, \hat{g}_{t, t+96}^{i}\right) \in \mathbb{R}^{96}, \quad \forall t \in\left\{1, \ldots, \theta^{i}\right\}, \quad \forall i \in I, \\
& \hat{d}_{t}^{i}=\left(\hat{d}_{t, t+1}^{i}, \ldots, \hat{d}_{t, t+96}^{i}\right) \in \mathbb{R}^{96}, \quad \forall t \in\left\{1, \ldots, \theta^{i}\right\}, \quad \forall i \in I,
\end{aligned}
$$

and sequences of historical forecasts for all sites $i \in I$

$$
\begin{aligned}
& \hat{g}^{i}=\left(\hat{g}_{1}^{i}, \ldots, \hat{g}_{\theta^{i}}^{i}\right) \in \mathbb{R}^{96 \times \theta^{i}}, \quad \forall i \in I, \\
& \hat{d}^{i}=\left(\hat{d}_{1}^{i}, \ldots, \hat{d}_{\theta^{i}}^{i}\right) \in \mathbb{R}^{96 \times \theta^{i}}, \quad \forall i \in I .
\end{aligned}
$$

The forecasting method employed by Schneider Electric combines auto-regressive models with random forests, and is inspired by the top-level methods used in GEFCom2014 [12].

By combining the chronicles (1) of historical observations with the chronicles (3) of historical forecasts, we can thus closely reproduce the information available to an online microgrid controller operating a real site. 


\subsection{Illustrating how sites differ in terms of predictability}

The dataset covers a large spectrum of situations regarding variability and predictability. To assess the predictability of the data at a given site $i \in I$, we first introduce the historical net demand observations

$$
z_{t}^{i}=d_{t}^{i}-g_{t}^{i} \in \mathbb{R}, \forall t \in\left\{1, \ldots, \theta^{i}\right\}, \forall i \in I,
$$

deduced from (1), and the historical net demand forecasts

$$
\hat{z}_{t}^{i}=\hat{d}_{t}^{i}-\hat{g}_{t}^{i} \in \mathbb{R}^{96}, \forall t \in\left\{1, \ldots, \theta^{i}\right\}, \forall i \in I,
$$

deduced from (3). Second, we normalize the historical net demand observations by

$$
\begin{aligned}
& \widetilde{z}_{t}^{i}=\frac{z_{t}^{i}-\underline{z}^{i}}{\bar{z}^{i}-\underline{z}^{i}} \in[0,1], \quad \forall t \in\left\{1, \ldots, \theta^{i}\right\}, \forall i \in I, \\
& \text { where }\left\{\begin{array}{l}
\bar{z}^{i}=\max _{1 \leq t \leq \theta^{i}}\left\{z_{t}^{i}\right\}, \quad \forall i \in I, \\
\underline{z}^{i}=\min _{1 \leq t \leq \theta^{i}}\left\{z_{t}^{i}\right\}, \quad \forall i \in I,
\end{array}\right.
\end{aligned}
$$

and we apply the same transformation to the components $\hat{z}_{t, t+1}^{i}, \ldots, \hat{z}_{t, t+96}^{i}$ of the historical net demand forecasts, yielding

$$
\widetilde{\hat{z}}_{t, t+k}^{i}=\frac{\hat{z}_{t, t+k}^{i}-\underline{z}^{i}}{\bar{z}^{i}-\underline{z}^{i}} \in \mathbb{R}, \forall k \in\{1, \ldots, 96\}, \forall t \in\left\{1, \ldots, \theta^{i}\right\}, \forall i \in I .
$$

Thus normalized, predictability can be compared across the pool of sites. Third, we define the Root Mean Square Error (RMSE) of the site $i \in I$ by

$$
\operatorname{RMSE}^{i}=\sqrt{\frac{1}{96 \times \theta^{i}} \sum_{t=1}^{\theta^{i}} \sum_{k=1}^{96}\left(\widetilde{\hat{z}}_{t, t+k}^{i}-\widetilde{z}_{t+k}^{i}\right)^{2}} .
$$

The diversity of the forecast error over the pool of 70 sites is shown in Figure 3 . Here, we ranked sites in increasing order of RMSE. We observe that the error is quite stable in the wide flat central part of sites distribution, except for the first $20 \%$ (with low forecast error) and for the last $20 \%$ (with high forecast error) of the sites. Thus, our dataset represents a diverse pool of microgrids, offering thus the possibility to assess the performance of microgrid management techniques on a large range of realistic industrial applications.

\section{The EMSx benchmark mathematical formulation}

After Schneider Electric's dataset presented in Sect. 2, the second component of the EMSx benchmark is a mathematical framework for the assessment of electric microgrid control techniques and algorithms. We formulate a benchmark problem to evaluate generic microgrid controllers in $\$ 3.1$. Then, we describe the structure of a controller, and how it is assessed by simulation along a partial chronicle in $\$ 3.2$. In $\$ 3.3$, we detail a score to compare the performance of controllers, and, in $\$ 3.4$, we extend it into a score to assess a whole controller-design technique. 




Figure 3: RMSE of the net demand forecast ( $Y$-axis) over the pool of 70 sites, ranked in increasing order of forecast error along the $X$-axis

\subsection{Microgrid control model}

We consider an electric microgrid composed of a photovoltaic power unit, an electric load and an energy storage system. We assume that all components of the microgrid share a single point of connection with the global grid. At this point, electric power can be imported or exported so as to satisfy the electric power demand (total load) at all times. We provide a schematic model of such a system in Figure 4.

We now introduce some notation. We represent the mathematical control of a microgrid over a finite number of discrete time steps $t \in\{0,1,2, \ldots, T-1, T\}$, where unit steps are spaced by $\Delta_{t}=15$ minutes. We denote a time interval between two decisions by $[t, t+1[$, and not by $[t, t+1]$, to indicate that a decision is taken at the beginning of the time interval $[t, t+1[$, and that a new one will be taken at the beginning of the time interval $[t+1, t+2[$, and that these two consecutive intervals do not overlap.

Storage dynamics. The storage system is assumed to be a lithium-ion battery (or a container of aggregated batteries), characterized by the coefficients $\left(c, \bar{l}, \rho_{c}, \rho_{d}\right)$ referring respectively to the battery's capacity $(\mathrm{kWh})$, maximum load $(\mathrm{kW})$, charge and discharge efficiency coefficients. The state of charge, at the beginning of the time interval $[t, t+1[$, is denoted by

$$
x_{t} \in[0,1] .
$$

The decision $u_{t}$, taken at the beginning of every time interval [t,t+1[, accounts for the energy charged $\left(u_{t} \geq 0\right)$ or discharged $\left(u_{t} \leq 0\right)$ during the time interval $[t, t+1[$. The dynamics of the state of charge is given by

$$
x_{t+1}=f\left(x_{t}, u_{t}\right), \forall t \in\{0, \ldots, T-1\},
$$






Figure 4: Schematic microgrid model

where the dynamics $f$ is given by

$$
f(x, u)=x+\frac{\rho_{c}}{c} u^{+}-\frac{1}{\rho_{d} c} u^{-}, \forall(x, u) \in[0,1] \times \mathbb{R},
$$

with $u^{+}=\max (0, u)$ and $u^{-}=\max (0,-u)$.

Constraints. Constraints of the form

$$
u_{t} \in \mathcal{U}\left(x_{t}\right), \forall t \in\{0, \ldots, T-1\}
$$

restrict decisions $u_{t}$ to the admissibility set (related to some of the battery parameters $\left(c, \bar{l}, \rho_{c}, \rho_{d}\right)$ by means of the dynamics $f$ in $(8)$ )

$$
\mathcal{U}(x)=\{u \in \mathbb{R} \mid \underline{u} \leq u \leq \bar{u} \text { and } 0 \leq f(x, u) \leq 1\},
$$

where $\bar{u}=\bar{l} \times \Delta_{t}$ and $\underline{u}=-\bar{l} \times \Delta_{t}$ are the bounds on the energy that can be exchanged with the battery during a 15 -minutes interval.

Uncertainties. For the purpose of defining the management costs, we introduce the uncertainties

$$
w_{t}=\left(g_{t}, d_{t}\right) \in \mathbb{R}^{2}, \forall t \in\{1, \ldots, T\}
$$

which represent a couple of photovoltaic generation $g_{t}$ and of energy demand $d_{t}$. As defined, uncertainties are exogenous model variables. When we turn to numerical experiments in $\$ 5$, sequences $\left(w_{1}, \ldots, w_{T}\right) \in \mathbb{R}^{2 \times T}$ of uncertainties are obtained as samples from the historical observations of $\$ 2.2$.

Costs. We now turn to the management costs. The stage cost during the time interval $[t, t+1[$ is

$$
L_{t}\left(u_{t}, w_{t+1}\right)=p_{t}^{+} \cdot e_{t+1}^{+}-p_{t}^{-} \cdot e_{t+1}^{-}, \forall t \in\{0, \ldots, T-1\}
$$


where

$$
e_{t+1}=d_{t+1}-g_{t+1}+u_{t}, \forall t \in\{0, \ldots, T-1\},
$$

is the energy exchanged with the grid - which, like the uncertainty $\left(g_{t+1}, d_{t+1}\right)$, materializes at the end of the time interval $\left[t, t+1\left[\right.\right.$, hence the index $t+1-$ and where $\left(p_{t}^{+}, p_{t}^{-}\right)$is the energy tariff (buying at price $p_{t}^{+}$and selling at price $p_{t}^{-}$) applied during the time interval $[t, t+1[$.

Given a sequence $\left(w_{1}, \ldots, w_{T}\right)$ of uncertainties and a sequence $\left(u_{0}, \ldots, u_{T-1}\right)$ of controls, we obtain the total operating cost

$$
\mathcal{L}\left(u_{0}, \ldots, u_{T-1}, w_{1}, \ldots, w_{T}\right)=\sum_{t=0}^{T-1} L_{t}\left(u_{t}, w_{t+1}\right) .
$$

In conclusion, we have introduced a dynamical system with dynamics (8), constraints (9) and a cost structure (11).

\subsection{Microgrid controller}

A microgrid controller is a mathematical device that, given some information at time $t$, yields a decision $u_{t}$. We now detail the structure of the controllers that we will consider. For this purpose, we introduce chronicles and management cost.

Partial chronicles. At the beginning of the time interval $[t, t+1[$, we may use all the past observations and past forecasts to make a decision $u_{t}$. For practical computational reasons, we have chosen to restrict this information to the partial observations

$$
\left(w_{t}, w_{t-1}, \ldots, w_{t-95}\right)=\left(\begin{array}{c}
g_{t}, \ldots, g_{t-95} \\
d_{t}, \ldots, d_{t-95}
\end{array}\right) \in \mathbb{R}^{2 \times 96}, \forall t \in\{0, \ldots, T-1\},
$$

of uncertainties 10 over the last 24 hours, and to the partial forecasts

$$
\left(\hat{w}_{t, t+1}, \ldots, \hat{w}_{t, t+96}\right)=\left(\begin{array}{c}
\hat{g}_{t, t+1}, \ldots, \hat{g}_{t, t+96} \\
\hat{d}_{t, t+1}, \ldots, \hat{d}_{t, t+96}
\end{array}\right) \in \mathbb{R}^{2 \times 96}, \forall t \in\{0, \ldots, T-1\},
$$

which represent a prediction of the uncertainties 10 for the next 24 hours. Combined together, we obtain the partial observations-forecasts

$$
\begin{aligned}
h_{t} & =\left(\begin{array}{c}
w_{t}, w_{t-1}, \ldots, w_{t-95} \\
\hat{w}_{t, t+1}, \ldots, \hat{w}_{t, t+96}
\end{array}\right) \in \mathbb{H}, \forall t \in\{0, \ldots, T-1\}, \\
\text { where } \quad \mathbb{H} & =\mathbb{R}^{2 \times 96} \times \mathbb{R}^{2 \times 96},
\end{aligned}
$$

and, stacking them all over the whole time span, we obtain the partial chronicle

$$
h=\left(h_{0}, \ldots, h_{T-1}\right) \in \mathbb{H}^{T} .
$$

Controller. A controller $\phi$ is a sequence of mappings

$$
\phi=\left(\phi_{0}, \ldots, \phi_{T-1}\right),
$$

where, for all $t \in\{0, \ldots, T-1\}$, we have $\phi_{t}:[0,1] \times \mathbb{H} \rightarrow \mathbb{R}$ with the constraints

$$
\phi_{t}\left(x_{t}, h_{t}\right) \in \mathcal{U}\left(x_{t}\right), \quad \forall\left(x_{t}, h_{t}\right) \in[0,1] \times \mathbb{H},
$$

where the constraint set $\mathcal{U}\left(x_{t}\right) \subset \mathbb{R}$ is defined in $9 \mathrm{~b}$. 
Management cost of a controller along a partial chronicle on a given site. All dynamics (8), constraints (9) and cost structure (11) depend on parameters relative to a site. Therefore, on a given site $i \in I$, we denote by $f^{i}$ the dynamics of the battery in (8) and by $\mathcal{U}^{i}$ the set-valued mapping defining the constraints as described in (9b), as they depend on the local parameters $\left(c^{i}, \bar{l}^{i}, \rho_{c}^{i}, \rho_{d}^{i}\right)$. We also denote by $L_{t}^{i}$ the stage cost in 11a - as it depends on the energy tariff $\left(p_{t}^{+, i}, p_{t}^{-, i}\right)$ which could possibly be local — and by $\mathcal{L}^{i}$ the total operating cost in (11c), as it depends on dynamics $f^{i}$, set-valued mappings $\mathcal{U}^{i}$ and stage costs $L_{t}^{i}$.

Besides battery parameters and energy tariffs, sites differ from each other in their historical data. For instance, the RMSE of the historical forecasts (Figure 3) varies across the pool of sites. Therefore, controllers in (13) may differ accordingly. This is why we denote by $\phi^{i}$ a controller for the site $i \in I$.

The application of a controller $\phi^{i}$ in 13 along a partial chronicle $h \in \mathbb{H}^{T}$ in $12 \mathrm{e}$ yields the management cost

$$
J^{i}\left(\phi^{i}, h\right)=\sum_{t=0}^{T-1} L_{t}^{i}\left(u_{t}^{i}, w_{t+1}\right),
$$

where, for all $t \in\{0, \ldots, T-1\}$, the uncertainty $w_{t+1}$ is a component of $h_{t+1}$, and the sequence $\left(u_{0}^{i}, \ldots, u_{T-1}^{i}\right)$ of controls is given by

$$
\begin{aligned}
x_{0}^{i} & =0, \\
x_{t+1}^{i} & =f^{i}\left(x_{t}^{i}, u_{t}^{i}\right), \\
u_{t}^{i} & =\phi_{t}^{i}\left(x_{t}^{i}, h_{t}\right) .
\end{aligned}
$$

The management cost $J^{i}\left(\phi^{i}, h\right)$ will serve the assessment of the controller $\phi^{i}$ in $\$ 3.3$.

\subsection{Designing and assessing a controller on a given site}

We consider a given site $i \in I$. We outline how to design and assess a controller $\phi^{i}$ as in (13).

Data partitioning. We have split the database in periods of one week ranging from Monday 00:00 to Sunday 23:45, each week containing thus $T=672=7 \times 24 \times 4$ time steps. With this, the database is now organized as a subset $\mathcal{D}^{i} \subset \mathbb{H}^{T}$ of chronicles, where $\mathbb{H}$ has been defined in 12 .

Then, we partition the chronicles in the data set $\mathcal{D}^{i}$ in two disjoint subsets, $\mathcal{C}^{i}$ for calibration (training, in-sample) and $\mathcal{S}^{i}$ for simulation (testing, out-of-sample):

$$
\mathcal{C}^{i} \cup \mathcal{S}^{i}=\mathcal{D}^{i} \subset \mathbb{H}^{T}, \quad \mathcal{C}^{i} \cap \mathcal{S}^{i}=\emptyset
$$

For the EMSx benchmark, we select randomly $40 \%$ of the weeks for simulation and let the other $60 \%$ be available for calibration.

Calibration data. The calibration data in $\mathrm{C}^{i}$ is available for the design of microgrid controllers as in (13). The design can result from any sort of technique (see examples in $\$ 5.1$ and in $\$ 5.2$ below). 
Simulation data. On top of the weekly periods, every simulation chronicle in $\mathcal{S}^{i}$ is augmented with the data of the Sunday before the period starts, following our definition 12e. Therefore, when simulating a microgrid controller, 24 hours of past history data is always available to the decision-maker. Simulation chronicles serve for testing only; as such, they cannot be employed for the design of a controller.

Parameters. Additionally, we have the battery parameters $\left(c^{i}, \bar{l}^{i}, \rho_{c}^{i}, \rho_{d}^{i}\right)$ (that have been adapted by Schneider Electric for the EMSx benchmark). For computing the management cost in (14), we use the energy tariff and time of use $\left(p_{t}^{+}, p_{t}^{-}\right)$, in $€ / \mathrm{kWh}$, from the French electricity provider Électricite de France (EDF); it is the same for all sites. At the beginning of any simulation, we assume the battery to be empty (i.e. $x_{0}=0$ ), and we do not impose any final cost (which is consistent with the expression $\mathcal{L}^{i}$ of the total operating cost in (11c)).

Lower bound for the management cost. For any controller $\phi^{i}$ as in 13 and any partial chronicle $h \in \mathbb{H}^{T}$ in $(12 \mathrm{e})$, the management cost $J^{i}\left(\phi^{i}, h\right)$ in (14) always has the so-called "anticipative" lower bound $\underline{J}^{i}(h)$, computed as the minimum of (14a) under the same constraints, initial state (14b) and dynamics (14c), but where the last constraint $u_{t}=\phi_{t}^{i}\left(x_{t}, h_{t}\right)$ in (14d) is now enlarged as $u_{t}=\psi_{t}^{i}\left(x_{t}, h\right)$, for all $t \in\{0, \ldots, T-1\}$, for any $\psi_{t}^{i}:[0,1] \times \mathbb{H}^{T} \rightarrow \mathbb{R}$. This gives a lower bound, because the minimization is done over "anticipative" control laws $\psi_{t}^{i}:[0,1] \times \mathbb{H}^{T} \rightarrow \mathbb{R}$, which encompass any controller $\phi^{i}$ as in $(13)$. Therefore, we easily get for any controller $\phi^{i}$, as in $(13)$, that we have

$$
\underline{J}^{i}(h) \leq J^{i}\left(\phi^{i}, h\right), \forall h \in \mathcal{S}^{i} .
$$

Performance score. With the battery parameters and energy tariff, and a given site controller $\phi^{i}$ as in (13), the simulator in (14) yields as many management costs $J^{i}\left(\phi^{i}, h\right)$ as there are chronicles $h$ in the simulation chronicles $\mathcal{S}^{i}$. Because the volume of energy production and consumption is variable from one site to another, raw management costs $J^{i}\left(\phi^{i}, h\right)$ are not suitable for a global performance analysis if we want to assess not only a given controller, but a controller-design technique (see examples in $\$ 5.1$ and in $\$ 5.2$, and see $\$ 3.4$ below).

Therefore, we shift the management cost of controller $\phi^{i}$ over a chronicle $h \in \mathcal{S}^{i}$ by subtracting the management cost of a dummy controller $\phi^{\mathrm{d}}$ such that $\phi_{t}^{\mathrm{d}}=0$, for $t \in\{0, \ldots, T-1\}$. This dummy zero policy $\phi^{\mathrm{d}}$ gives us a baseline operating cost, the one that we would get when the microgrid is not equipped with an EMS and a battery. After the shift, we define the gain of controller $\phi^{i}$ by the following expression

$$
G^{i}\left(\phi^{i}\right)=\frac{1}{\left|\mathcal{S}^{i}\right|} \sum_{h \in \mathcal{S}^{i}}\left(J^{i}\left(\phi^{\mathrm{d}}, h\right)-J^{i}\left(\phi^{i}, h\right)\right),
$$

which expresses the average gain of introducing $\phi^{i}$ in site $i$, over the baseline case of a dummy controller that does not use the battery. The lower bound for the management cost in (16) provides a natural upper bound for the gain

$$
\bar{G}^{i}=\frac{1}{\left|\mathcal{S}^{i}\right|} \sum_{h \in \mathcal{S}^{i}}\left(J^{i}\left(\phi^{\mathrm{d}}, h\right)-\underline{J}^{i}(h)\right),
$$

where $G^{i}\left(\phi^{i}\right) \leq \bar{G}^{i}$. 
In order to ease the performance analysis of a controller-design technique over an aggregated group of sites from $I$, we scale the gain of $\phi^{i}$ with the upper bound $\bar{G}^{i}$, and we define the performance score

$$
\mathcal{G}^{i}\left(\phi^{i}\right)=\frac{G^{i}\left(\phi^{i}\right)}{\bar{G}^{i}}
$$

The higher the score, the higher the gain allowed by the controller $\phi^{i}$. A controller $\phi^{i}$ that improves on the dummy controller $\phi^{\mathrm{d}}$ gives a score in $[0,1]$, else the score is negative.

\subsection{Assessing a controller-design technique}

Controller-design technique. In addition to assessing a given controller, we also aim at assessing a design technique for controllers. We provide examples of design techniques in 5.1 and in \$5.2. In particular, when the same design technique is used across all sites, we will consider a collection $\left\{\phi^{i}\right\}_{i \in I}$ of controllers derived from the application of a single design technique, but adapted to each site $i \in I$.

Performance score of a collection of controllers. For a given collection $\left\{\phi^{i}\right\}_{i \in I}$ of controllers, one per site, we average the performance score $(19)$ over sites, yielding the performance score (of a collection $\left\{\phi^{i}\right\}_{i \in I}$ of controllers)

$$
\mathcal{G}\left(\left\{\phi^{i}\right\}_{i \in I}\right)=\frac{1}{|I|} \sum_{i \in I} \mathcal{G}^{i}\left(\phi^{i}\right) .
$$

When the controllers $\phi^{i}$ in the collection $\left\{\phi^{i}\right\}_{i \in I}$ have been designed by the same technique, the score $\mathcal{G}\left(\left\{\phi^{i}\right\}_{i \in I}\right)$ in 20 is a proxy to measure the performance of a controller-design technique over a large range of situations, both in time of the year and in type of microgrid. It permits an immediate interpretation for practitioners interested in deploying a technique to design controllers on real microgrids. The higher the score, the higher the gain allowed by the technique.

\section{The EMSx benchmark software}

After Schneider Electric's dataset presented in Sect. 2, and the mathematical framework presented in Sect. 3, the third component of the EMSx benchmark is the EMSx benchmark software, that we have developed and implemented as a Julia [4] package named EMSx.j1.

\subsection{Requirements for the EMSx benchmark software}

Performing a simulation loop over the 70 sites $i \in I$, for computing the management costs (14) of a collection $\left\{\phi^{i}\right\}_{i \in I}$ of controllers on the simulation chronicles of $\left\{\mathcal{S}^{i}\right\}_{i \in I}$, and finally obtaining the score (20), is a time-consuming computing task. Indeed, our total pool of simulation chronicles $\left\{\mathcal{S}^{i}\right\}_{i \in I}$ gathers data from 2474 testing weeks, each of which requires 672 (7 days in a week $\times 96$ decisions in a day) calls to a controller, hence a total of 1.6 million calls for assessing a single control technique.

We have developed a software, called EMSx.j1, to ease the numerical assessment of controllers in the context of the EMSx benchmark. Our first goal is to provide an efficient and 
fast computing tool for running every simulation loop. We have chosen the Julia language, as it is a perfect candidate for processing the large amount of data made available in the EMSx benchmark dataset. In particular, Julia makes it simple to distribute the simulation loop on several CPU cores, which enables us to release the EMSx.j1 package with parallel computing options. Moreover, Julia meets our second expectation, namely that our simulation software should be flexible enough to easily implement a large range of controllers as defined in $\$ 3.2$. We illustrate such flexibility in Sect. 5. where we outline the numerical results obtained with EMSx.jl for various controller design techniques.

\subsection{What the EMSx.jl software package does}

Given a site $i \in I$, a controller $\phi^{i}$ in (13) and a partial chronicle $h \in \mathbb{H}^{T}$ in (12e) (in practice, $h \in \mathcal{S}^{i}$, the simulation chronicles in (15)), the EMSx. $j 1$ software returns the sequence of states of charge of the battery, the stagewise costs, and, above all, the management cost $J^{i}\left(\phi^{i}, h\right)$ in (14) and the corresponding computing time. To this end, a EMSx.jl user must provide the implementation of her controller $\phi^{i}$ in Julia code following an API that we briefly describe now.

Figure 5 illustrates how the dummy controller $\phi_{t}^{\mathrm{d}}=0, t \in\{0, \ldots, T-1\}$ can be implemented and tested in a few lines of code. First, we define a new type for our controller, named DummyController, as a subtype of a built-in EMSx.jl type, named AbstractController (line 3). Then, we implement the body of the compute_control function (in Julia jargon, method) for the new specialized DummyController type (line 5). In the given example, the body is reduced to a simple zero expression, as we have to implement a method which always returns zero. Eventually, we create an instance of a DummyController (line 8) and launch the simulation over all simulation chronicles (line 10) passing the created instance as argument. Battery parameters $\left(c, \bar{l}, \rho_{c}, \rho_{d}\right)$ (referred to as metadata, line 13) and energy tariff $\left(p^{+}, p^{-}\right)$(line 12) can be changed by the user to run a custom simulation.



Figure 5: Example of the implementation and simulation of the dummy controller with the EMSx.jl package

We can implement more complex examples by using the information object given in the input arguments of the compute_control function. Figure 6 displays the fields of the Information type, an EMSx.jl built-in type. An instance of this type gives access to the 
running time step $t \in\{0, \ldots, T-1\}$ (line 2), to the state of charge $x_{t}$ in (8a) (line 3), and to the content of the partial observations-forecasts $h_{t}$ in (12) (line 4-7). This online information allows us to define a controller $\phi$ as in (13). Besides, the Information type has fields providing access to the energy price $\left(p^{+}, p^{-}\right)$(line 8), to the battery parameters $\left(c, \bar{l}, \rho_{c}, \rho_{d}\right)$ (line 9$)$ and to the site reference $i \in I$ (line 10), which let us define a specific controller $\phi^{i}$ for the site $i \in I$. All-in-all, beyond our toy example of Figure 5, line 5-6, the information object in the input argument of the compute_control function enables the implementation of more sophisticated controllers, such as the ones that we introduce and evaluate in $\$ 5$.



Figure 6: Detail of the Information type from the EMSx. jl package

As an example of using the Information type, a controller that empties half of the battery would be implemented as in Figure 5, with the lines 5-6 replaced by

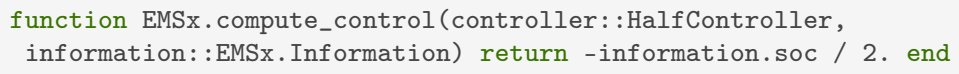

The code of EMSx.jl and more illustrative controller examples are publicly available at https://github.com/adrien-le-franc/EMSx.jl.

\section{$5 \quad$ Numerical experiments}

To illustrate how we can use the EMSx controller benchmark of Sect. 3, we present several controllers and provide their scores. These controllers $\phi$ all share the same structure: for any step $t \in\{0, \ldots, T-1\}$, the quantity $\phi_{t}\left(x_{t}, h_{t}\right)$ in 13 is not given by an analytical formula, but as the solution of a reference optimization problem. Controller-design techniques differ according to the nature of this latter problem, which is solved at every step $t \in\{0, \ldots, T-1\}$, that is, online ("on the fly") as a function of the current available quantities $\left(x_{t}, h_{t}\right)$, namely state of charge of the battery and couples of observations-forecasts up to time $t$ (see (12e) A comprehensive overview of such techniques and algorithms is given in [2].

Before we start, we introduce some terminology. First, we define a scenario as a sequence $\left\{w_{t}\right\}_{t \in \mathcal{T}}$ of uncertainties $w_{t}=\left(g_{t}, d_{t}\right)$ as in $(10)$, and where $\mathcal{T} \subseteq\{1, \ldots, T\}$. Second, we stress the difference between open-loop and closed-loop solutions and optimization problems. In an intertemporal optimization problem, one may look either for solutions that are only functions of time (open-loop), or for solutions that are functions of time and of other variables that 
are available up to this time (closed-loop). Therefore, an optimization problem is said to be open-loop (resp. closed-loop) when its solutions are open-loop (resp. closed-loop). We refer to [5, §1.1.3] for a discussion about the difference between open-loop and closed-loop controls.

In $\$ 5.1$, we present a class of so-called lookahead methods where the reference optimization problem is multistage open-loop. In contrast, in $\$ 5.2$, we present a class of so-called cost-to-go methods where the reference optimization problem, solved online, is one-stage but depends on cost-to-go functions which, themselves, are the output of closed-loop optimization problems which are computed offline. In $\$ 5.3$, we comment the results obtained when applying the controllers above on the EMSx controller benchmark.

\subsection{Controllers obtained by lookahead computation methods}

In lookahead methods, one solves, for every step $t \in\{0, \ldots, T-1\}$, a reference multistage ("looking ahead" from the current step $t$ ) optimization problem which is open-loop, be it deterministic (MPC) or stochastic (OLFC). Stochastic (scenario-based) lookahead methods are limited by the exponential growth of the computing time with respect to the number of scenarios.

\subsubsection{Model Predictive Control}

The Model Predictive Control (MPC) method is one of the most famous lookahead techniques [2, Vol.1, §6.1]. The MPC method mainly exploits the forecast data. It yields a controller $\phi^{\mathrm{MPC}}=\left(\phi_{0}^{\mathrm{MPC}}, \ldots, \phi_{T-1}^{\mathrm{MPC}}\right)$ by solving a sequence of multistage deterministic optimization problems over a fixed ${ }^{1}$ horizon $H$ (in the numerical application, $H=96$ )

$$
\begin{cases}u_{t}^{\star} \in \underset{u_{t}}{\arg \min } \min _{\left(u_{t+1}, \ldots, u_{t+H-1}\right)} & \sum_{s=t}^{t+H-1} L_{s}\left(u_{s}, \hat{w}_{t, s+1}\right), \\ & x_{s+1}=f\left(x_{s}, u_{s}\right), \forall s \in\{t, \ldots, t+H-1\}, \\ & u_{s} \in \mathcal{U}\left(x_{s}\right), \forall s \in\{t, \ldots, t+H-1\}, \\ \phi_{t}^{\mathrm{MPC}}\left(x_{t}, h_{t}\right)=u_{t}^{\star}, & \end{cases}
$$

where only the first value $u_{t}^{\star}$ of an optimal sequence $\left(u_{t}^{\star}, u_{t+1}^{\star}, \ldots, u_{t+H-1}^{\star}\right)$ is kept.

When used in simulation, only the simulation (testing) chronicles in $\mathcal{S}^{i}$ in the partition (15) are used in the reference optimization problem (21), and not the calibration (training) chronicles in $\mathrm{C}^{i}$; moreover, only a subvector (of available forecasted values) of the whole vector (12) of partial observations-forecasts is used, namely $\left(\hat{w}_{t, t+1}, \ldots, \hat{w}_{t, t+H-1}\right)$.

Due to the mathematical expressions for the dynamics (8), the constraints $(9)$ and the cost function (11), the multistage deterministic optimization problem (21) formulates here as a linear program.

\subsubsection{Open Loop Feedback Control}

The Open Loop Feedback Control (OLFC) method belongs to the family of online lookahead methods and its approach is similar to MPC, but for a stochastic component. It yields a

\footnotetext{
${ }^{1}$ When the horizon extends further than the period, we truncate the lookahead window to $\min (H, T-t+1)$.
} 
controller $\phi^{\mathrm{OLFC}}=\left(\phi_{0}^{\mathrm{OLFC}}, \ldots, \phi_{T-1}^{\mathrm{OLFC}}\right)$ by a sequence of multistage open-loop stochastic optimization problems over a fixed ${ }^{1}$ horizon $H$ (in the numerical application, $H=96$ )

$$
\begin{cases}u_{t}^{\star} \in \underset{u_{t}}{\arg \min } \min _{\left(u_{t+1}, \ldots, u_{t+H-1}\right)} & \sum_{\sigma \in \mathbb{S}} \pi_{t}^{\sigma}\left(\sum_{s=t}^{t+H-1} L_{s}\left(u_{s}, w_{t, s+1}^{\sigma}\right)\right), \\ & x_{s+1}=f\left(x_{s}, u_{s}\right), \forall s \in\{t, \ldots, t+H-1\}, \\ & u_{s} \in \mathcal{U}\left(x_{s}\right), \forall s \in\{t, \ldots, t+H-1\}, \\ \phi_{t}^{\mathrm{OLFC}}\left(x_{t}, h_{t}\right)=u_{t}^{\star} . & \end{cases}
$$

The reference optimization problem 22 is open-loop because the sequence of control variables $\left(u_{t+1}, \ldots, u_{t+H-1}\right)$ in the (second) $\min$ is not indexed by the scenario references $\sigma \in \mathbb{S}$. Only the first value $u_{t}^{\star}$ of an optimal sequence $\left(u_{t}^{\star}, u_{t+1}^{\star}, \ldots, u_{t+H-1}^{\star}\right)$ is kept.

The reference optimization problem 22 is stochastic because of the scenarios $\left(w_{t, t+1}^{\sigma}\right.$, $\left.\ldots, w_{t, t+96}^{\sigma}\right)_{\sigma \in \mathbb{S}}$, together with their probabilities $\left(\pi_{t}^{\sigma}\right)_{\sigma \in \mathbb{S}}$. These scenarios and their probabilities are built from two sources: on the one hand, from the subvector $\left(\hat{w}_{t, t+1}, \ldots, \hat{w}_{t, t+H-1}\right)$ of available forecasted values given in the whole vector (12) of partial observations-forecasts in the simulation (testing) chronicles in $\mathcal{S}^{i}$ in (15); on the other hand, from partial observationsforecasts in the calibration (training) chronicles in $\mathrm{C}^{i}$ in $(15)$, from which we calibrate a scenario generation model. In the forthcoming numerical experiments in $\$ 5.3$, we generate scenarios by modeling the deviations from the net demand 24-hour forecast as a Markov chain. We detail our scenario generation method in the Appendix (\$ number of samples used varies between 10,50 or 100 scenarios.

\subsection{Controllers obtained by cost-to-go computation methods}

In cost-to-go methods, one solves online, for every step $t \in\{0, \ldots, T-1\}$, a reference single stage stochastic optimization problem, which itself depends on cost-to-go functions, computed offline. These functions are called cost-to-go because, ideally, they map any state of the system to the optimal, over strategies (closed-loop), expected future cost from a given time step to the final horizon. The Stochastic Dynamic Programming (SDP) method is the most famous of cost-to-go computation techniques [2]. Cost-to-go methods are limited by the exponential growth of the computing time with respect to the dimension of the state space. In SDP methods, we use random variables, defined on an abstract probability space $(\Omega, \mathcal{F}, \mathbb{P})$, and designed by bold capital letters like $\mathbf{W}$.

\subsubsection{Stochastic Dynamic Programming}

Whereas MPC and OLFC are based on an open-loop reference optimization problem, Stochastic Dynamic Programming is based on a closed-loop reference optimization problem, like in 24 .

- In the offline phase of the SDP algorithm, one computes a sequence of so-called value functions $\left(V_{t}\right)_{t=0,1, \ldots, T-1, T}$ by the Bellman (or dynamic programming) equation, backward for $t \in\{0, \ldots, T-1\}$,

$$
\begin{aligned}
& V_{T}(x)=0 \\
& V_{t}(x)=\min _{u \in \mathcal{U}(x)} \sum_{\sigma \in \mathbb{S}_{\text {off }}} \pi_{t+1}^{\text {off }, \sigma}\left(L_{t}\left(u, w_{t+1}^{\text {off }, \sigma}\right)+V_{t+1}(f(x, u))\right) .
\end{aligned}
$$


- In the online phase of the SDP algorithm, one computes

$$
\left\{\begin{array}{l}
u_{t}^{\star} \in \underset{u \in \mathcal{U}\left(x_{t}\right)}{\arg \min } \sum_{\sigma \in \mathbb{S}_{\text {on }}} \pi_{t+1}^{\mathrm{on}, \sigma}\left(L_{t}\left(u, w_{t+1}^{\mathrm{on}, \sigma}\right)+V_{t+1}\left(f\left(x_{t}, u\right)\right)\right) \\
\phi_{t}^{\mathrm{SDP}}\left(x_{t}, h_{t}\right)=u_{t}^{\star} .
\end{array}\right.
$$

The reference optimization problem (23a) $-23 \mathrm{~b}$ is stochastic because of the scenarios $\left(w_{t+1}^{\text {off }, \sigma}\right)_{\sigma \in \mathbb{S}^{\text {off }}}$, together with their probabilities $\left(\pi_{t+1}^{\mathrm{off}, \sigma}\right)_{\sigma \in \mathbb{S}^{\text {off }}}$, and of the scenarios $\left(w_{t+1}^{\mathrm{on}, \sigma}\right)_{\sigma \in \mathbb{S} \text { on }}$, together with their probabilities $\left(\pi_{t+1}^{\text {on }, \sigma}\right)_{\sigma \in \mathbb{S}^{\circ} \text {. }}$.

The scenarios indexed by $\sigma \in \mathbb{S}^{\text {off }}$, and their probabilities, are built exclusively from partial observations-forecasts in the calibration (training) chronicles in $\mathrm{C}^{i}$, in (15). The scenarios indexed by $\sigma \in \mathbb{S}^{\text {on }}$, and their probabilities, could additionally integrate partial observationsforecasts in the simulation (testing) chronicles in $\mathcal{S}^{i}$ in (15). The reader will find details of our scenario generation method in Appendix $\$$ A.2.

The reference optimization problem (23a)-23b is closed-loop because, under proper assumptions, it provides the optimal solution to the following multistage stochastic optimization problem (where the minimum is over strategies $\psi$ which depend both on time and on past uncertainties as in (24d)

$$
\begin{aligned}
\min _{\psi} & \mathbb{E}\left[\sum_{t=0}^{T-1} L_{t}\left(\mathbf{U}_{t}, \mathbf{W}_{t+1}\right)\right], \\
& \mathbf{X}_{t+1}=f\left(\mathbf{X}_{t}, \mathbf{U}_{t}\right), \forall t \in\{0, \ldots, T-1\}, \\
& \mathbf{X}_{0}=x_{0}, \\
& \mathbf{U}_{t}=\psi_{t}\left(\mathbf{W}_{0}, \ldots, \mathbf{W}_{t}\right), \forall t \in\{0, \ldots, T-1\} \\
& \mathbf{U}_{t} \in \mathcal{U}\left(\mathbf{X}_{t}\right), \forall t \in\{0, \ldots, T-1\} .
\end{aligned}
$$

Problem (24) is optimally solved by the Bellman equation (23a) in the case where the random variables $\left(\mathbf{W}_{0}, \ldots, \mathbf{W}_{T}\right)$ (noise process) are stagewise independent [2,5].

\subsubsection{SDP-AR}

To account for possible stagewise dependence in the uncertainties (noise process in (24)), one can extend the state space with the observations of the $i$-th net demand lags $z_{t-i}=$ $d_{t-i}-g_{t-i}, i=1, \ldots, k$. This gives a new state $\widetilde{x}_{t}-$ which is a compression of the information given by $\left(x_{t}, h_{t}\right)$ - and new elements of a model, like in $\$ 3.1$, with a new dynamics $\widetilde{f}_{t}$, new constraints $\widetilde{\mathcal{U}}$, and a new stage cost $\widetilde{L}_{t}$ as follows, for all $t \in\{0, \ldots, T-1\}$ :

$$
\begin{aligned}
\widetilde{x}_{t} & =\left(x_{t}, z_{t}, \ldots, z_{t-k+1}\right) \in[0,1] \times \mathbb{R}^{k}, \\
\widetilde{x}_{t+1} & =\widetilde{f}_{t}\left(\widetilde{x}, u_{t}, \epsilon_{t+1}\right), \\
\widetilde{f}_{t}\left(\widetilde{x}_{t}, u_{t}, \epsilon_{t+1}\right) & =\left(\begin{array}{c}
f\left(x_{t}, u_{t}\right) \\
\sum_{j=0, \ldots, k-1} \alpha_{t}^{j} z_{t-j}+\beta_{t}+\epsilon_{t+1} \\
z_{t}, \ldots, z_{t-k+2}
\end{array}\right), \\
\widetilde{\mathcal{U}}\left(\widetilde{x}_{t}\right) & =\mathcal{U}\left(x_{t}\right), \\
\widetilde{L}_{t}\left(\widetilde{x}_{t}, u_{t}, \epsilon_{t+1}\right) & =L_{t}\left(u_{t}, \sum_{j=0, \ldots, k-1} \alpha_{t}^{j} z_{t-j}+\beta_{t}+\epsilon_{t+1}\right) .
\end{aligned}
$$


The coefficients $\alpha_{t}^{0}, \ldots, \alpha_{t}^{k-1}$ and the additive terms $\beta_{t}$ are the elements of an auto-regressive model of order $k$ (noted $\mathrm{AR}(k)$ )

$$
\mathbf{Z}_{t+1}=\sum_{j=0, \ldots, k-1} \alpha_{t}^{j} \mathbf{Z}_{t-j}+\beta_{t}+\boldsymbol{\epsilon}_{t+1}, \quad \forall t \in\{0, \ldots, T-1\}
$$

for the net demand process $\mathbf{Z}$. When the error process $\boldsymbol{\epsilon}$ is assumed to be stagewise independent, the following algorithm provides an optimal solution to the multistage stochastic optimization problem 24.

- In the offline phase of the SDP-AR algorithm, one computes a sequence of new value functions $\left(\widetilde{V}_{t}\right)_{t=0, \ldots, T}$, backward for $t \in\{0, \ldots, T-1\}$, by

$$
\begin{aligned}
& \widetilde{V}_{T}(\widetilde{x})=0, \\
& \widetilde{V}_{t}(\widetilde{x})=\min _{u \in \widetilde{\mathcal{U}}(\widetilde{x})} \sum_{\sigma \in \mathbb{S}^{\circ} \text { off }} \pi_{t+1}^{\text {off }, \sigma}\left(\widetilde{L}_{t}\left(\widetilde{x}, u, \epsilon_{t+1}^{\text {off }, \sigma}\right)+\widetilde{V}_{t+1}\left(\widetilde{f}_{t}\left(\widetilde{x}, u, \epsilon_{t+1}^{\mathrm{off}, \sigma}\right)\right)\right) .
\end{aligned}
$$

- In the online phase of the SDP-AR algorithm, one computes

$$
\left\{\begin{array}{l}
u_{t}^{\star} \in \underset{u \in \widetilde{\mathcal{U}}\left(\widetilde{x}_{t}\right)}{\arg \min } \sum_{\sigma \in \mathbb{S}^{\text {on }}} \pi_{t+1}^{\mathrm{on}, \sigma}\left(\widetilde{L}_{t}\left(\widetilde{x}_{t}, u, \epsilon_{t+1}^{\mathrm{on}, \sigma}\right)+\widetilde{V}_{t+1}\left(\widetilde{f}_{t}\left(\widetilde{x}_{t}, u, \epsilon_{t+1}^{\mathrm{on}, \sigma}\right)\right)\right) \\
\phi_{t}^{\mathrm{SDP}-\mathrm{AR}}\left(x_{t}, h_{t}\right)=u_{t}^{\star} .
\end{array}\right.
$$

\subsection{Using EMSx to compare controller-design techniques}

We now comment the results obtained when applying the controller-design techniques introduced in $\$ 5.1$ and in $\$ 5.2$ to the EMSx benchmark. Numerical experiments were run on an Intel Core Processor of $2.5 \mathrm{GHz}$ with $22 \mathrm{~GB}$ RAM. We used the LP solver CPLEX 12.9 for MPC, OLFC and to compute the lower bounds $\underline{J}^{i}(h), h \in \mathcal{S}^{i}, i \in I$, in (16). We have summarized our results in Table 1 .

Our goal is to illustrate how EMSx enables a fine comparison of controller-design techniques. First, we focus on lookahead methods in \$5.3.1, second, we turn to cost-to-go methods in $\$ 5.3 .2$, and finally, we give a comparative analysis of the winner control techniques from both family of methods in $\$ 5.3 .3$. We conclude that, among our pool of candidate techniques, SDP-AR stands out as the best microgrid control method on the EMSx benchmark.

In what follows, we organize the discussion around three points: i) performance scores $(20)$ (second column of Table 1); ii) (relative) gains $2^{2}$ (17) disaggregated per site (Figures 7 and 8); iii) CPU time performances. Regarding CPU time, we report the online time (fourth column of Table 1) as the average computing time required to yield a single control $u_{t}$, and the offline time (third column of Table 1) as the average computing time that is to be spent prior to the simulation step for solving the online control problems defined in (21), (22), (23b) and (26b).

\subsubsection{Lookahead methods}

First, we examine performance scores (second column of Table 1). Whereas MPC uses a single scenario (the forecast), OLFC uses multiple scenarios; this helps OLFC improving the

\footnotetext{
${ }^{2}$ We recall that gains were defined relatively to the cost performance of a dummy controller.
} 


\begin{tabular}{l|lll} 
& $\begin{array}{c}\text { Performance } \\
\text { score }\end{array}$ & $\begin{array}{c}\text { Offline time } \\
\text { (seconds) }\end{array}$ & $\begin{array}{c}\text { Online time } \\
\text { (seconds) }\end{array}$ \\
\hline MPC & 0.487 & - & $9.8210^{-4}$ \\
\hline OLFC-10 & 0.506 & - & $1.1410^{-2}$ \\
\hline OLFC-50 & 0.513 & - & $8.6210^{-2}$ \\
\hline OLFC-100 & 0.510 & - & $1.8710^{-1}$ \\
\hline \hline SDP & 0.691 & 2.67 & $3.0910^{-4}$ \\
\hline SDP-AR(1) & 0.794 & 38.1 & $4.4410^{-4}$ \\
\hline SDP-AR(2) & 0.795 & 468 & $5.5510^{-4}$ \\
\hline Upper bound & $\mathbf{1 . 0}$ & - & - \\
\hline
\end{tabular}

Table 1: Scores (second column, the higher the better) $\mathcal{G}\left(\left\{\phi^{i}\right\}_{i \in I}\right)$ in 20 and time performances (third and fourth column, the lower the better) for collections $\left\{\phi^{i}\right\}_{i \in I}$ of controllers $\phi^{i}$ designed with techniques (first column) from $\$ 5.1$ and $\$ 5.2$ on the EMSx benchmark. The symbol - indicates an irrelevant item

average score of MPC from 0.487 to 0.513 . As expected, increasing the number of scenarios from 10 to 50 improves the score of OLFC. However, the performance remains stagnant when pushing up to 100 scenarios, with a slight decrease of OLFC-100 to 0.510. We expect that the improvement between MPC and OLFC should be sensitive to the scenario generation method employed. With our method, the progress of OLFC is modest.

Second, we turn to appraise the gains disaggregated per site. In Figures 7 and 8 , we display the gains per site $G^{i}\left(\phi^{i}\right)$ in (17). We omitted OLFC-10 and OLFC-100 to improve readability, given that these methods perform slightly worse than OLFC-50. We observe that OLFC-50 returns slightly higher gains than MPC for 58 out of 70 sites. We conclude that the stochastic approach of OLFC makes it a slightly more accurate microgrid controller-design technique than MPC.

Third, we discuss the computing time. Lookahead methods advantageously do not include an offline stage (third column of Table 1). However, except for MPC, they require a rather long online computing time (fourth column of Table 1), with an order of magnitude between $10^{-4}$ and $10^{-1}$ seconds per call to the controller, for they call a LP solver at each time step. We observe that OLFC is at least about ten times slower than MPC. As expected, the more we add sample scenarios, the longer the OLFC computing time. We observe that improving the amount of scenarios from 50 to 100 doubles the online time. Even though the online time of OLFC-100 is still reasonable for a field implementation of a microgrid controller, it took us about 46 hours to run the simulation over the 2474 simulation weeks. Despite the much longer computing time, OLFC-100 did not return higher gains than OLFC-50. For this reason, we did not consider more than 100 generated scenarios.

\subsubsection{Cost-to-go methods}

First, we examine performance scores (second column of Table 1). We see that a plain SDP controller yields scores jumping to 0.691. The results of SDP can be improved up to 0.794 in the SDP-AR formulation. However, We observe that the gain from extending the lag of an $\mathrm{AR}(1)$ model to an $\mathrm{AR}(2)$ model is almost null. Therefore, we do not report the gains of SDP-AR (2) in Figures 7 and 8. 




Figure 7: Gain $G^{i}\left(\phi^{i}\right)$ ( $Y$-axis) in (17) per sites $i \in I$ ( $Y$-axis) of MPC, OLFC-50, SDP and SDP-AR (1); sites are ranked in increasing order of RMSE along the $X$-axis

Second, we turn to appraise the gains disaggregated per site. Looking closer at the per site performances, SDP-AR (1) outperforms SDP for 69 of the 70 sites. As suggested by its performance score, Figure 8 reveals that the gains allowed by SDP-AR (1) are close to the upper bounds $\bar{G}^{i}, i \in I$ in $18 \mathrm{a}$.

Third, we discuss the computing time. Cost-to-go methods display fast online times (fourth column of Table 1), with an order of magnitude between $10^{-3}$ and $10^{-4}$ seconds per call to the controller. However, cost-to-go methods require offline CPU time (third column of Table 1 ) for computing value functions. The complexity of SDP is well known for growing exponentially with the state space, which is well illustrated by our results: from SDP to SDP-AR (1) to SDP-AR (2), we add one dimension to the state space at each improvement of the method, which multiplies by a factor of 10 the offline time. Given the low improvement of gain from SDP-AR (1) to SDP-AR (2), we find the offline time of the latter method dissuasive. Finally, SDP-AR (1) appear as the best trade-off between computing time and cost performance.

\subsubsection{A comparison between lookahead and cost-to-go methods}

We now discuss the comparative performances of OLFC-50 and SDP-AR (1). Both techniques represent the best candidate of its family of method.

Cost performance. First, we examine performance scores (second column of Table 1). The main observation is that the performance score of SDP-AR (1) is more than $50 \%$ higher than the one of OLFC-50 (second column of Table 1). 




Figure 8: Gains ( $Y$-axis) per sites $i \in I$ ( $X$-axis): the upper bound (dashed line) is the quantity $\bar{G}^{i}$ in $18 \mathrm{a}$; the horizontal dotted and dashed line represents the null gain of the dummy controller; the other symbols represent the quantities $G^{i}\left(\phi^{i}\right)$ in (17) corresponding to the four methods SDP-AR (1), SDP, OLFC-50, MPC; sites are ranked in increasing order of gain $\bar{G}^{i}$ along the $X$-axis 
Second, we turn to appraise the gains disaggregated per site. The observed aggregated dominance is confirmed when looking closer at the per site performances in Figures 7 and 8. Indeed, we see that SDP-AR(1) outperforms OLFC-50 for 68 of the total pool of 70 sites. Even more, these two figures reveal that lookahead methods lag behind cost-to-go ones for almost all sites, and that the gap can be significant on a few outlying sites. The underperformance of OLFC-50 on these sites explains the score gaps of Table 1.

Third, we discuss the relationship between the cost performances of both techniques and the predictability of the sites, measured by the RMSE (7). For this purpose, we comment and detail Figure 9. We immediately observe that, for SDP-AR (1), there is no strong link between cost performance and predictability. Regarding OLFC-50, a statistical analysis reveals that the correlation between the RMSE value and the OLFC-50 score is moderate, with a Pearson correlation coefficient of -0.54 (the lower the RMSE, the higher the score). For most of the sites, the RMSE explains very little of the performance of OLFC-50, to the point that OLFC50 performs poorly on some sites with low RMSE values (and thus high forecast accuracy). Now, we focus on a few number of sites that appear as outliers: Site 33 and Site 59 (the two circles at the far right), and Site 48 (the circle at the top left). OLFC-50 achieves its highest scores 0.965 on Site 33 and 0.910 on Site 59, which both have very regular and easily predictable load profiles, and its worse score -0.340 on Site 48 , the least predictable site of the pool, with a RMSE of 0.17. Notice that this negative score of -0.340 means that OLFC-50 performs worse on average than the dummy controller $\phi^{\mathrm{d}}$. In contrast, we observe that SDPAR (1) scores 0.566 on Site 48, which highlights the robustness of the cost-to-go methods for the management of a microgrid in a highly unpredictable context.

Computing time performance. Second, we discuss the computing time. On the one hand, regarding offline time, SDP-AR (1) cannot do better than OLFC-50, obviously, but the average offline time of SDP-AR (1) (38.1 seconds) is reasonable for a field implementation of a microgrid controller. On the other hand, regarding online time, OLFC-50 is not as good as SDP-AR (1) - with an average online time of OLFC-50 about 100 times longer than the one of SDP-AR (1) - but its order of magnitude $\left(10^{-2}\right.$ seconds) remains acceptable. Thus, all in all, computing time is not a discriminating point between the two techniques.

\section{Conclusion}

We have introduced EMSx, an Energy Management System benchmark to compare electric microgrid controllers, hence controller-design techniques. EMSx is made of three key components. The dataset provided by Schneider Electric ensures a diverse pool of realistic microgrids with photovoltaic power integration. The mathematical framework is explicit. The simulation code is accessible in the EMSx. $\mathrm{jl}$ package, designed to welcome various sorts of control algorithms. All components of the benchmark are publicly available, so that other researchers willing to test controllers on EMSx may reproduce experiments easily.

Regarding our numerical results, we observe a gap between cost-to-go methods and lookahead methods. The SDP-AR (1) controller-design technique stands out as the best tradeoff between cost optimality and computing time performance. However, there is a range of possible improvements to explore. Among interesting directions, other scenario generation techniques could be tested to see how does the OLFC controller react. Beyond plain score improvements, enriching contributions could arise from the reduction of the computing time, or 




Figure 9: Performance score $\mathcal{G}^{i}\left(\phi^{i}\right)$ per sites $i \in I$ for the SDP-AR (1) and OLFC-50 computation methods ( $X$-axis) versus RMSE ( $Y$-axis); the vertical line recalls the baseline score positioning of a dummy controller

from changing the performance metrics (for instance with the use of risk measures), and from further comparative analysis of methods, especially to better explain the gap between lookahead and cost-to-go methods. We are also looking forward to controllers inspired from other research fields than multistage deterministic or stochastic optimization, including heuristics, robust optimization and reinforcement learning.

Acknowledgements We thank Efficacity and Schneider Electric for the PhD funding of Adrien Le Franc. Additionally, we are grateful for the feedbacks and data supply from Peter Pflaum and Claude Le Pape (Schneider Electric) and we thank our colleague Tristan Rigaut (Efficacity) for insightful tips about the Julia language. We thank the Guest Editor and the Reviewers for their insightful comments that helped improve the manuscript.

\section{A Scenario generation}

\section{A.1 Generation of scenarios for the Open Loop Feedback Control algo- rithm}

Our scenario generation method is inspired by 20,21, which address such generation for so-called day-ahead energy management problems. We use a simplified model for tractable generation adapted to dynamical control. Since uncertainties $w_{t}=\left(g_{t}, d_{t}\right)$ are directly plugged in the cost (11a) as the net demand $z_{t}=d_{t}-g_{t}$, we compress the generation of uncertainty scenarios $\left(w_{t, t+1}^{\sigma}, \ldots, w_{t, t+96}^{\sigma}\right) \in \mathbb{R}^{2 \times 96}$ in 22 to the generation of net demand scenarios 
$\left(z_{t, t+1}^{\sigma}, \ldots, z_{t, t+96}^{\sigma}\right) \in \mathbb{R}^{96}$. Following the original methods, we choose day part separators to reduce the 96 dimensional vector to a few skeleton points for scenario construction (intermediate values are linearly interpolated). We choose to concentrate on the forecast error at $t+15$ minutes, $t+1$ hour, $t+2$ hours, $t+4$ hours, $t+12$ hours and $t+24$ hours, so that our model only samples values of $z_{t, t+j}^{\sigma}$ for $j \in\{1,4,8,16,48,96\}$. We select 10 relevant values of the net demand error at each separator $j \in\{1,4,8,16,48,96\}$ by applying the $K$-means algorithm [6, §13] on the historical error $z_{t+j}^{i}-\hat{z}_{t, t+j}^{i}$, combining the historical observations (1) and forecasts (3) of the calibration data of the Site $i \in I$ considered. Then, we compute $10 \times 10$ transition matrices for the error between consecutive separators. With this model, we are able to sample net demand scenarios $\left(z_{t, t+1}^{\sigma}, \ldots, z_{t, t+96}^{\sigma}\right)$ given a single value forecast and to compute their probabilities $\pi_{t}^{\sigma}$. Separate probability distributions of the initial error at $t+1$ are calibrated depending on the time of the day, and on whether the initial time step $t$ corresponds to a week day or a weekend day. We alleviate computing costs by reusing transition matrices regardless of the initial value of $t$. However we calibrate separate matrices for week days and weekend days. Figure 10 provides examples of scenarios generated with our method.



Figure 10: Example of scenarios generated from a 24 hours net demand forecast

\section{A.2 Generation of scenarios for the SDP and SDP-AR algorithms}

For SDP methods, we choose to discretize each dimension of the state space in 10 values, whereas the control space is restricted to 20 values and the noise space to 10 values. Since uncertainties $w_{t}=\left(g_{t}, d_{t}\right)$ are directly plugged in the cost 11a as the net demand $z_{t}=d_{t}-g_{t}$, we compress the calibration of the distributions of $\left(\mathbf{W}_{1}, \ldots, \mathbf{W}_{T}\right)$ to the calibration of the distributions of $\left(\mathbf{Z}_{1}, \ldots, \mathbf{Z}_{T}\right)$. We use the $K$-means algorithm to fit discrete probabilities $\pi_{t+1}^{\text {off }, \sigma}, \pi_{t+1}^{\text {on, } \sigma}$ on the historical observations $\left(g^{i}, d^{i}\right)$ (1) of the calibration data of the Site $i \in I$ considered. These discrete distributions serve the computation of expectations in (23a)-23b). While we could leverage the data available on the fly in the online phase, we use the same probability distributions in the offline phase and in the online phase. Separate distributions (of $\mathbf{Z}_{t}$ ) are 
calibrated depending on the time of the day and on whether the time step $t+1$ corresponds to a week day or a weekend day. We compute one value function per site for the horizon of one week. In the SDP-AR formulation, we calibrate the $\mathrm{AR}(k)$ models using least square regression and calibrate distributions of the residual error $\left(\boldsymbol{\epsilon}_{t}\right)$ with the same approach as for $\mathbf{Z}_{t}$.

\section{References}

[1] D. P. Bertsekas. Dynamic Programming and Optimal Control: Approximate Dynamic Programming. Athena Scientific, fourth edition, 2012.

[2] Dimitri P Bertsekas. Dynamic programming and optimal control, volume 1. Athena scientific Belmont, MA, 1995.

[3] Dimitri P Bertsekas. Dynamic programming and suboptimal control: A survey from ADP to MPC. European Journal of Control, 11(4-5):310-334, 2005.

[4] Jeff Bezanson, Stefan Karpinski, Viral B Shah, and Alan Edelman. Julia: A fast dynamic language for technical computing. arXiv preprint arXiv:1209.5145, 2012.

[5] Pierre Carpentier, Jean-Philippe Chancelier, Guy Cohen, and Michel De Lara. Stochastic multi-stage optimization. In Probability Theory and Stochastic Modelling, volume 75. Springer, 2015.

[6] Jerome Friedman, Trevor Hastie, and Robert Tibshirani. The elements of statistical learning. Springer series in statistics New York, 2001.

[7] Carlos E. Garcia, David M. Prett, and Manfred Morari. Model predictive control: theory and practice - a survey. Automatica, 25(3):335-348, 1989.

[8] Felix A Gers, Nicol N Schraudolph, and Jürgen Schmidhuber. Learning precise timing with lstm recurrent networks. Journal of machine learning research, 3(Aug):115-143, 2002.

[9] Pierre Haessig, Thibaut Kovaltchouk, Bernard Multon, Hamid Ben Ahmed, and Stéphane Lascaud. Computing an optimal control policy for an energy storage. arXiv preprint arXiv:1404.6389, 2014.

[10] Faeza Hafiz, MA Awal, Anderson Rodrigo de Queiroz, and Iqbal Husain. Real-time stochastic optimization of energy storage management using rolling horizon forecasts for residential pv applications. In 2019 IEEE Industry Applications Society Annual Meeting, pages 1-9. IEEE, 2019.

[11] Benjamin Heymann, J Frédéric Bonnans, Francisco Silva, and Guillermo Jimenez. A stochastic continuous time model for microgrid energy management. In 2016 European Control Conference (ECC), pages 2084-2089. IEEE, 2016.

[12] Tao Hong, Pierre Pinson, Shu Fan, Hamidreza Zareipour, Alberto Troccoli, and Rob J Hyndman. Probabilistic energy forecasting: Global energy forecasting competition 2014 and beyond, 2016. 
[13] Phillip Oliver Kriett and Matteo Salani. Optimal control of a residential microgrid. Energy, 42(1):321-330, 2012.

[14] Nils Lohndorf and Alexander Shapiro. Modeling time-dependent randomness in stochastic dual dynamic programming. European Journal of Operational Research, 273(2):650-661, 2019.

[15] Daniel E Olivares, Ali Mehrizi-Sani, Amir H Etemadi, Claudio A Cañizares, Reza Iravani, Mehrdad Kazerani, Amir H Hajimiragha, Oriol Gomis-Bellmunt, Maryam Saeedifard, Rodrigo Palma-Behnke, et al. Trends in microgrid control. IEEE Transactions on smart grid, 5(4):1905-1919, 2014.

[16] Martin L. Puterman. Markov Decision Processes: Discrete Stochastic Dynamic Programming. John Wiley \& Sons, Inc., 1st edition, 1994.

[17] Tristan Rigaut, Pierre Carpentier, Jean Philippe Chancelier, Michel De Lara, and Julien Waeytens. Stochastic optimization of braking energy storage and ventilation in a subway station. IEEE Transactions on Power Systems, 34(2):1256-1263, 2018.

[18] Alexander Shapiro. Analysis of stochastic dual dynamic programming method. European Journal of Operational Research, 209(1):63-72, 2011.

[19] Alexander Shapiro, Darinka Dentcheva, and Andrzej Ruszczynski. Lectures on Stochastic Programming: Modeling and Theory, Second Edition. Society for Industrial and Applied Mathematics, Philadelphia, PA, USA, 2014.

[20] Andrea Staid, Jean-Paul Watson, Roger J-B Wets, and David L Woodruff. Generating short-term probabilistic wind power scenarios via nonparametric forecast error density estimators. Wind Energy, 20(12):1911-1925, 2017.

[21] David L Woodruff, Julio Deride, Andrea Staid, Jean-Paul Watson, Gerrit Slevogt, and César Silva-Monroy. Constructing probabilistic scenarios for wide-area solar power generation. Solar Energy, 160:153-167, 2018. 